\title{
Modélisation et analyse expérimentale du procédé de soudage par friction
}

\author{
Mohamed Guidara, Fakhreddine Dammak et Abderrazak Dhiab ${ }^{a}$ \\ Laboratoire des Systèmes Électromécaniques (LASEM), ENIS, BP W 3038, Sfax, Tunisie
}

Reçu le 22 janvier 2003, accepté le 8 juillet 2005

\begin{abstract}
Résumé - Cette étude contribue à la modélisation numérique du soudage par friction pour l'identification des paramètres de soudage. La modélisation repose sur le calcul de la quantité de chaleur générée par frottement et sur la répartition spatiale de la chaleur en tenant compte de la variation des propriétés thermiques et physiques du matériau. Des essais de soudage par friction nous ont permis d'étudier l'effet du temps de friction sur la propagation de la chaleur et sur la taille de la zone affectée thermiquement.
\end{abstract}

Mots clés : Soudage par friction / éléments finis / couplage thermomécanique

Abstract - Modelling and experimental analysis of the welding process by friction. Our study contributes to a numerical modelling of friction welding and identification of welding parameters. The modelling is based on evaluation of heat quantity generated by friction as well as on the spatial distribution of the heat with taking into account of the variation of the thermal and physical properties of the material. Friction welding tests allow to study the effect of the friction time both on heat propagation as on thermally affected zone size.

Key words: Welding by friction / finite element / thermo mechanical coupling

\section{Introduction}

Depuis 1945, le soudage par friction est utilisé pour les matières thermoplastiques. Pour souder des métaux par ce procédé des tentatives ont été faites peu après 1940 par Klopostock, mais ce n'est qu'en 1956 que les Russes Chudickov et Vill ont mis au point une méthode pratique de soudage des métaux par friction. Plusieurs travaux concernent la technologie du procédé de soudage par friction et l'étude de l'influence de divers paramètres sur la qualité de la soudure comme Hollander [1], Laour [2], Dufin [3], Sayegh [4] et Ayadi [5]. La mise en ouvre du procédé de soudage par friction présente toujours un certain nombre d'inconnues en raison de la limitation de la maîtrise des paramètres influents et très complexes tels que le temps de friction, le temps de forgeage, la pression de friction, la pression de forgeage etc. Des travaux d'optimisation ont été publiés par Cazes [6] et Primot [7]. Différents auteurs comme Rykalin, Rich et Roberts et Cheng avant 1981 ont modélisé l'élévation de température générée à l'interface par friction par des méthodes analytiques. Le problème de couplage thermomécanique a

\footnotetext{
a Auteur correspondant : dhiebabd@yahoo.fr
}

été traité en hyperélasticié par Miehe [8] et Armero et Simo [9], en plasticité par Simo et Miehe [10], en viscoplasticité par Argyris et al. [11] et en endommagement élastoplastique par Srikanth et Zabaras [12]. La modélisation numérique et la simulation du phénomène de soudage par friction par l'introduction des principales propriétés thermomécaniques du matériau à souder ont permis à des auteurs d'optimiser quelques paramètres de soudage. Une modélisation correcte de l'opération de soudage par friction nécessite la formulation d'un certain nombre de problèmes que nous présentons selon l'ordre d'importance :

- couplage thermomécanique à haute température,

- contact entre les pièces à souder,

- changement de phase du matériau à souder.

Dans cette étude, seul le problème du couplage thermomécanique est traité. Nous présentons ici un résumé de la formulation du couplage thermique, le calcul de la déformation plastique résultante et la méthode d'intégration de ce problème non-linéaire. La validation du modèle numérique est confrontée aux résultats expérimentaux obtenus sur une machine de soudage par friction pilotée. 


\section{Nomenclature}

\begin{tabular}{|ll|}
\hline$J$ & Jacobien \\
$F$ & Gradient de déformation \\
$\boldsymbol{F}_{v}$ & Vecteur des forces volumiques \\
$\tau$ & Tenseur de contraintes de Kirchhoff \\
$c$ & Chaleur spécifique \\
$q$ & Flux de chaleur \\
$R$ & Source de chaleur \\
$D_{\mathrm{m}}$ & Dissipation mécanique \\
$H$ & Terme de couplage \\
$\kappa$ & Coefficient de conduction thermique \\
$\Theta$ & Température absolue \\
$H$ & Potentiel d'échauffement structural élastique \\
$\Psi_{\mathrm{vol}}$ & Énergie libre volumique \\
$\Psi_{\text {iso }}$ & Énergie libre isochorique \\
$T$ & Énergie thermique \\
$M$ & Énergie thermo-volumique \\
$\psi^{p}$ & Énergie plastique \\
$p$ & Déformation plastique équivalente \\
$K$ & Module de compressibilité \\
$\alpha$ & Coefficient de dilatation \\
$\mu$ & Module de cisaillement \\
$T(\Theta)$ & Potentiel purement thermique \\
$M(J, \Theta)$ & Terme de couplage thermomécanique \\
$\psi_{\text {iso }}\left(\overline{\boldsymbol{b}}^{\mathrm{e}}\right)$ & Terme de la partie isochorique \\
$\psi_{\mathrm{vol}}(J)$ & Terme de la partie volumique \\
$\delta \mathbf{u}$ & Vecteur déplacement virtuel \\
$\tau_{\text {iso }}$ & Tenseur de contraintes de Kirchhoff isochorique \\
$G_{\mathrm{ext}}$ & Travail virtuel des forces extérieures \\
$G_{\mathrm{int}}$ & Travail virtuel des forces intérieures \\
$\mu(r)$ & Coefficient de frottement \\
$P(r)$ & Pression de forgeage \\
$\omega$ & Vitesse angulaire de rotation \\
$A_{\mathrm{cl}}$ & Premier point de transformation lors du chauffage \\
$A_{\mathrm{c} 3}$ & Troisième point de transformation lors du chauffage \\
$A_{\mathrm{r} 1}$ & Premier point de transformation lors du refroidissement \\
$A_{\mathrm{r} 3}$ & Troisième point de transformation lors du refroidissement \\
&
\end{tabular}

\section{Modélisation}

Dans la littérature, il existe deux méthodes de résolution des équations de couplage thermomécanique. La première approche est basée sur une discrétisation temporelle globale couplée à une discrétisation spatiale avec des éléments finis spécifiques pour obtenir finalement un système d'équations algébriques couplées, Oden [13]. Cette méthode est connue sous le nom de «stratégie de la solution simultanée ». Dans la deuxième approche, connue sous le nom de «stratégie chancelée », le système est découpé selon les différents champs couplés. Dans le cas du couplage thermomécanique, cette méthode est associée à une partition en une phase de déformation mécanique isotherme, et une phase de conduction thermique en l'absence de déformation, Argyris [11].

Malgré une grande stabilité numérique, la première approche présente l'inconvénient de conduire à résoudre un système généralement non symétrique de très grande taille. La stratégie chancelée permet de surmonter ces inconvénients mais souvent au détriment de la stabilité numérique. Ce problème de stabilité a été résolu par Armero et Simo [9] qui établissent un schéma de fractionnement du pas constitué d'une phase de déformation mécanique adiabatique suivie d'une phase de conduction thermique sans déformation. En réalité, la diminue de la stabilité numérique de la méthode de la stratégie chancelée n'est pas significative dans la plupart des cas de mise en forme des matériaux, Miehe [8].

La réponse thermomécanique d'un solide subissant des déformations plastiques est gouvernée par les équations de conservation de la quantité de mouvement et d'énergie couplées aux relations constitutives et aux conditions aux limites appropriées. Ces équations de conservation permettent d'avoir les expressions locales des équations d'équilibre mécanique et de la chaleur. L'équation d'équilibre locale dans la configuration courante s'écrit sous la forme proposée par Simo et Miehe [10]

$$
J \operatorname{div}(\boldsymbol{\tau} / J)+\boldsymbol{F}_{v}=\mathbf{0}
$$

où $J=\operatorname{det}(\boldsymbol{F})$ est le déterminant du gradient de déformation $F$ et $J$ est le Jacobien, $\boldsymbol{\tau}$ est le tenseur des 
contraintes de Kirchhoff et $\boldsymbol{F}_{v}$ est le vecteur des forces volumiques. L'équation de conservation de l'énergie, ou équation de la chaleur a pour configuration générale :

$$
c \dot{\Theta}=-J \operatorname{div}(\boldsymbol{q} / J)+R+D_{\mathrm{m}}-H
$$

où $\Theta$ est la température absolue, $R$ est la source de chaleur, $D_{\mathrm{m}}$ est la dissipation mécanique, $H$ est un terme de couplage qui représente l'échauffement structural et $\boldsymbol{q}$ est le vecteur flux de chaleur donné par la loi de Fourier :

$$
\mathbf{q}=-k \operatorname{grad}(\Theta)
$$

où $k$ est le coefficient de conduction thermique. Considérons un matériau hyper-élastique compressible, avec sa fonction énergie libre découplée en parties volumique $\psi_{\mathrm{vol}}$, isochorique $\psi_{\text {iso }}$, thermique $T$, thermovolumique $M$ et plastique $\psi^{\mathrm{p}}$ :

$$
\psi=\psi_{\mathrm{vol}}(J)+\psi_{\text {iso }}\left(\overline{\boldsymbol{b}}^{\mathrm{e}}\right)+T(\Theta)+M(J, \Theta)+\psi^{\mathrm{p}}(p, \Theta)
$$

où $p$ est la déformation plastique équivalente, $\overrightarrow{\boldsymbol{b}}=$ $J^{-2 / 3} \boldsymbol{b}^{\mathrm{e}}, \psi_{\mathrm{vol}}(J)$ est la partie volumique, $\psi_{\text {iso }}\left(\overline{\boldsymbol{b}}^{\mathrm{e}}\right)$ est la partie isochorique, $T(\Theta)$ est le potentiel purement thermique et $M(J, \Theta)$ est le terme de couplage thermomécanique dû à l'expansion thermique qui permet l'obtention du potentiel d'échauffement structural élastique $H$. Ces fonctions sont :

$$
\begin{aligned}
\psi_{\mathrm{vol}}(J) & =\frac{K}{2}(\log J)^{2}, \\
\psi_{\text {iso }}\left(\overline{\boldsymbol{b}}^{\mathrm{e}}\right) & =\frac{1}{2} \mu\left(\operatorname{tr}\left(\overline{\boldsymbol{b}}^{e}\right)-3\right) \\
T(\Theta) & =c\left[\left(\Theta-\Theta_{0}\right)-\Theta \log \left(\Theta / \Theta_{0}\right)\right], \\
M(J, \Theta) & =\left(\Theta-\Theta_{0}\right) G(J), \\
G(J) & =-3 \alpha J \psi_{\mathrm{vol}}^{\prime}(J)
\end{aligned}
$$

où $K$ est le module de compressibilité, $\mu$ est le module de cisaillement, $c$ est la chaleur spécifique et $\alpha$ est le coefficient de dilatation. À partir de cette expression de l'énergie libre, le tenseur de contraintes de Kirchhoff et le module tangent peuvent être déduits selon Miehe [8]. Pour le comportement élastoplastique nous utilisons le critère d'écoulement de Von Mises avec un écrouissage isotrope. L'intégration des équations associées à ce modèle, sont données en Annexe A.

La forme réduite de l'équation d'équilibre mécanique avec un modèle mixte en pression et dilatation s'écrit sous la forme :

$$
\begin{array}{r}
G=\int_{V} \nabla \delta \mathbf{u}:\left(\boldsymbol{\tau}_{\text {iso }}+J \bar{p} \mathbf{1}\right) \mathrm{d} V+\int_{V} \delta \bar{p}(J-\bar{J}) \mathrm{d} V \\
+\int_{V} \delta \bar{J}\left(-p+\psi^{\prime}(\bar{J})\right) \mathrm{d} V-G_{\text {ext }}(\delta \boldsymbol{u})=0
\end{array}
$$

La discrétisation par éléments finis est réalisée avec des éléments bilinéaires axisymétriques à quatre nœuds.
Le même élément de référence est utilisé pour la discrétisation spatiale de l'équation de la chaleur dont la forme intégrale et générale est :

$$
\begin{aligned}
G= & \int_{V} \delta \Theta\left(c \dot{\Theta}+J \operatorname{div}(\boldsymbol{q} / J)-D_{\mathrm{m}}+H\right) \mathrm{d} V \\
& -G_{\text {ext }}(\delta \Theta)=0 \\
G_{\text {int }}= & \int_{V} \delta \Theta\left(c \dot{\Theta}-D_{\mathrm{m}}+H\right) \mathrm{d} V-\int_{V} \nabla \delta \Theta \cdot \boldsymbol{q} \mathrm{d} V
\end{aligned}
$$

Un schéma d'intégration implicite de type Euler est choisi pour la discrétisation temporelle. La résolution numérique du problème couplé est alors réalisée en deux étapes : résolution du problème mécanique suivi d'une correction thermique. La première étape correspond à la résolution de l'équation de la quantité de mouvement dans des conditions isothermes. La deuxième étape correspond à la résolution de l'équation de la chaleur dans une configuration figée. Pour la résolution de ces deux équations, toutes les constantes du matériau sont considérées comme des fonctions de la température.

\section{Dispositif expérimental}

La machine de soudage par friction pilotée automatiquement, conçue et réalisée par nos soins, est représentée sur la figure 1 . Le cycle de soudage est représenté sur la figure 2 .

Le cycle de soudage par friction commence par :

- la première séquence est le rapprochement des deux pièces à souder (Fig. 3a) ;

- la deuxième séquence est la phase d'accostage avec une faible force axiale pour obtenir la friction entre les deux pièces à souder (Fig. 3b);

- la troisième séquence est la phase d'échauffement, la température s'élève de l'ordre de $920^{\circ} \mathrm{C}$ (Fig. 3c);

- la quatrième séquence l'arrêt total de la partie tournante avec une force axiale supérieure à celle de la phase d'accostage correspond à : c'est la phase de forgeage (Fig. 3d) ;

- la cinquième séquence donne la fin de la phase de forgeage (Fig. 3e) ;

- la dernière séquence montre la vue de la pièce après soudage (Fig. 3f).

Les séquences du cycle de soudage sont illustrées sur les figures $3 \mathrm{a}-\mathrm{f}$.

\section{Résultats expérimentaux numériques}

La validation du modèle est réalisée en confrontant les résultats expérimentaux à ceux obtenus par l'étude numérique. En effet, on peut distinguer deux types de résultats :

- les mesures après soudage renseignent sur l'état final de la soudure; 


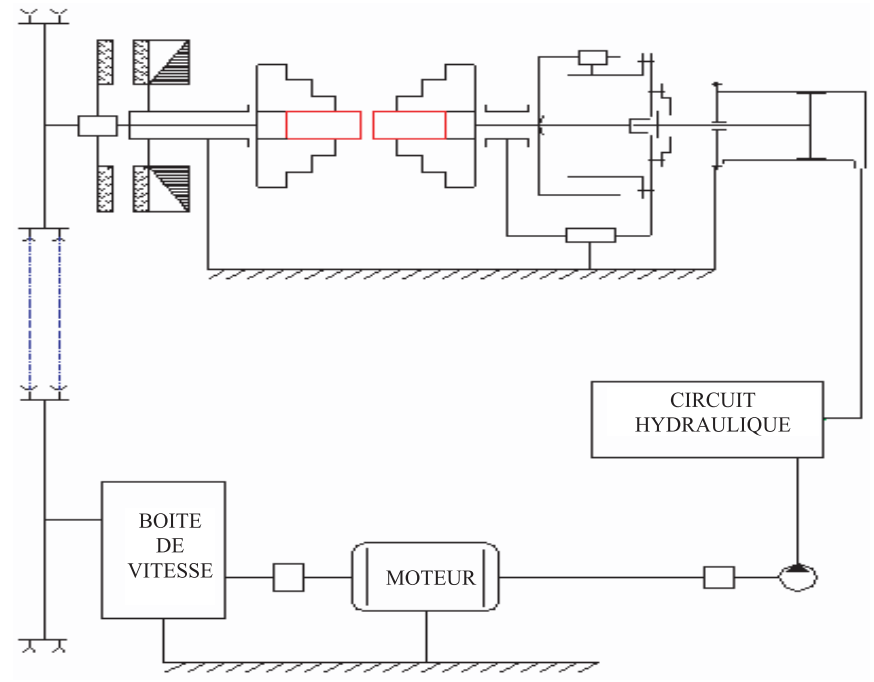

Fig. 1. Schéma cinématique de la machine de soudage par friction.

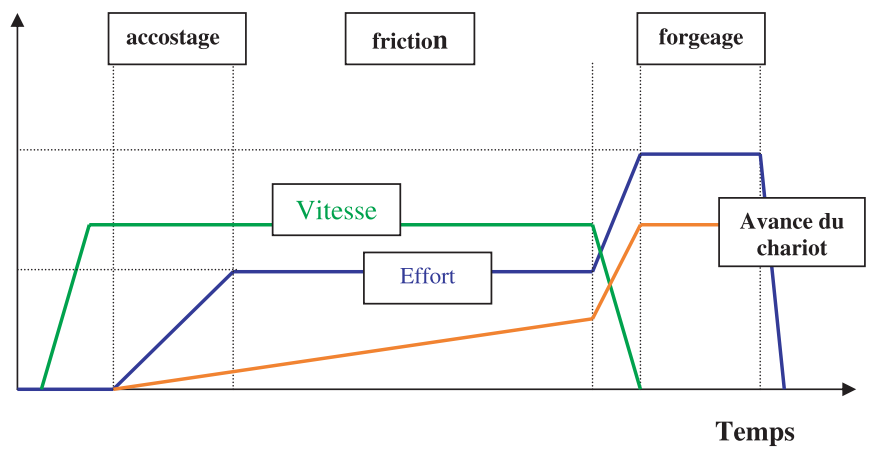

Fig. 2. Séquences d'un cycle de soudage.

- les mesures en temps réel pendant le soudage, assez riches en informations mais exigeant une instrumentation et un outil informatique pour traiter et enregistrer les données mesurées. Dans le cas du soudage par friction de deux aciers l'acier C45 et l'acier doux, les caractéristiques mécaniques sont regroupées dans le tableau 1.

$E$ : module de Young, $R_{\mathrm{e}}$ : limite élastique, $R_{\mathrm{m}}$ : résistance mécanique, $K$ et $n$ : paramètres de Ludwik et $A \%$ : allongement en pourcent.

Ces deux matériaux ont été retenus pour la soudure par friction. Ils sont de types industriels : l'acier doux est réputé par sa ductilité et est applicable pour les arbres de transmission, l'acier C45 est moins ductile que l'acier doux mais il est connu pour la fabrication des pignons.

Nous avons choisi les paramètres d'essais suivants :

- Vitesse de frottement : $80 \mathrm{~m} \cdot \mathrm{min}^{-1}$

- Temps de friction : $t_{\mathrm{c}}=2 \mathrm{~s}$

- Temps de forgeage $: t_{\mathrm{f}}=1 \mathrm{~s}$

- Flux de chaleur : $q=11$ W.mm ${ }^{-2}$

- Pression de forgeage : $P_{\mathrm{f}}=100 \mathrm{MPa}$

- Coefficient de frottement $: \mu_{\text {moy }}=0,17$

- Rayon moyen de l'éprouvette $R_{\text {moy }}=5 \mathrm{~mm}$

- Pression moyenne de friction : $P_{\mathrm{c}}=50 \mathrm{MPa}$
- Longueur des éprouvettes : $L=50 \mathrm{~mm}$.

La modélisation numérique de couplage thermique de deux solides en mouvement relatif repose sur la connaissance de la quantité de chaleur générée par frottement. Le flux de chaleur correspondant est supposé réparti uniformément de façon symétrique et opposé aux deux pièces. Ce flux $q$, donné par l'équation (8), est considéré constant pendant la phase de friction. Dès l'arrêt de la partie tournante, le flux devient nul

$$
q=2 \omega \int_{0}^{R} \mu(r) P(r) \mathrm{d} r
$$

où $\omega$ étant la vitesse de rotation, $\mu(r)$ étant le coefficient de frottement et $P(r)$ étant la pression de forgeage.

\subsection{Conditions aux limites et maillage}

Dans le cas de deux matériaux similaires, on peut tenir compte de la symétrie, en limitant la modélisation à une seule pièce et en introduisant un modèle de contact rigide/déformable (phase de friction). Dans le cas de deux matériaux différents (géométrie et/ou matériaux), il faut réaliser la discrétisation du problème sur les deux pièces en introduisant un modèle de contact entre deux corps déformables.

Les conditions aux limites du problème sont données sur la figure 4a dans le repère axisymétrique $(r, z)$. La figure $4 \mathrm{~b}$ présente le maillage réalisé sur la pièce à étudier. Un maillage raffiné est réalisé à l'extrémité OD de la pièce à souder, car cette zone est l'objet de très grandes modifications thermiques et mécaniques.

\subsection{Position d'une isotherme}

Lorsqu'une soudure est réalisée sans déplacement de la source de chaleur, il n'y a pas d'état quasi-stationnaire car la réalisation de la soudure est due à un apport d'énergie localisée. Celle-ci est assez intense et momentanée et n'est pas compensée par l'écoulement calorifique au sein des pièces assemblées. Pour atteindre une température maximale de forgeage à l'interface, nous avons utilisé un temps de friction de deux secondes. Après cette période, la phase de forgeage commence. La température continue à augmenter de part et d'autre de l'interface malgré une décroissance à l'interface. On constate que la température dans certains points continue à augmenter même si la génération de chaleur par friction est stoppée. Les courbes de température le long de l'axe $z$ après la phase de friction atteignent les températures maximales en chaque point (Figs. 5, 6) après un certain retard.

\subsection{Cycle thermique}

Granjon [14] a défini le cycle thermique du soudage par friction par une loi de variation de la température 


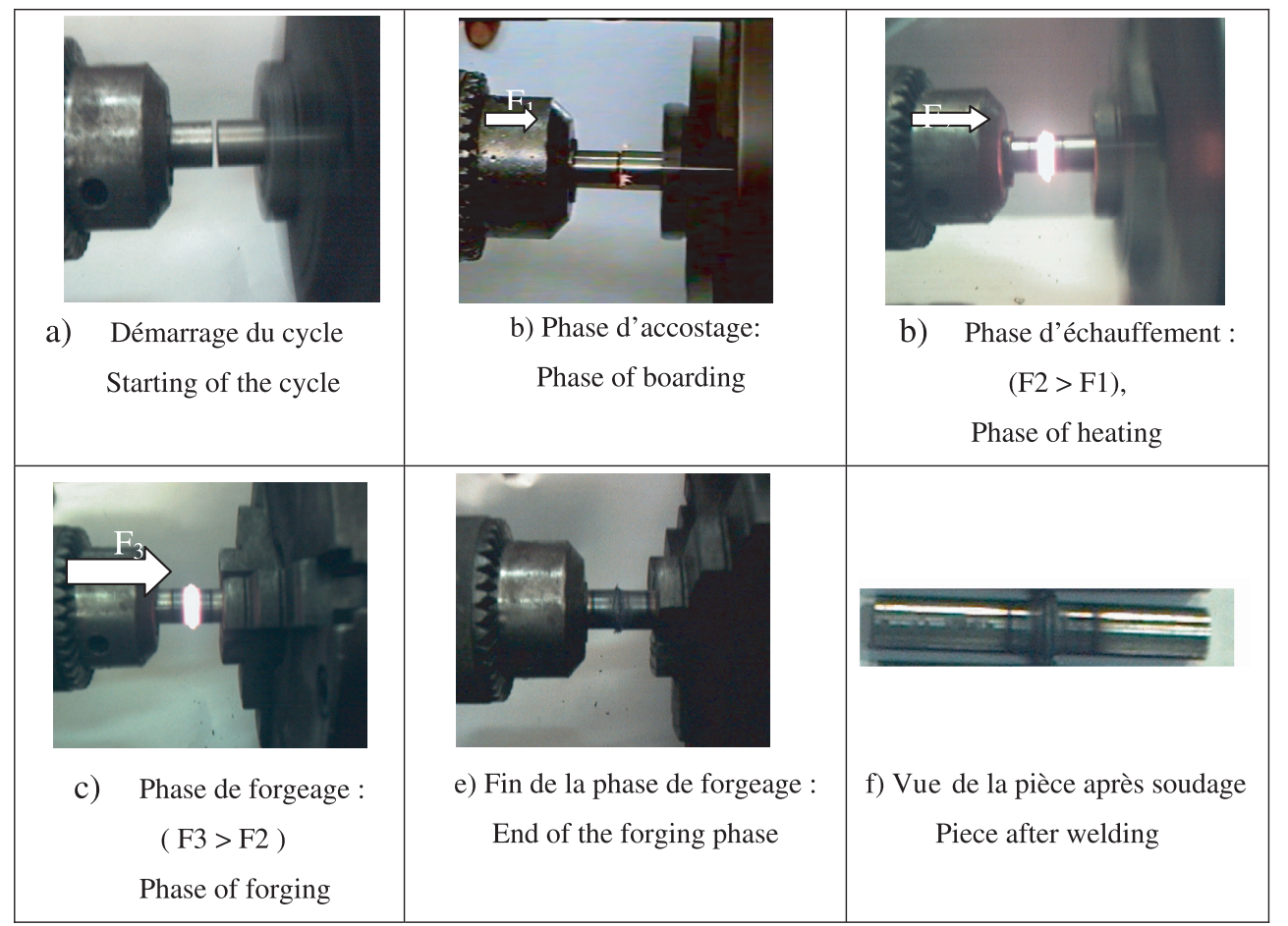

Fig. 3. Cycle de soudage.

Tableau 1. Propriétés des matériaux étudiés en statique.

\begin{tabular}{lcccccc}
\hline Matériaux & $E(\mathrm{MPa})$ & $R_{\mathrm{e}}(\mathrm{MPa})$ & $R_{\mathrm{m}}(\mathrm{MPa})$ & $A \%$ & $K(\mathrm{MPa})$ & $n$ \\
\hline C45 & 207000 & 320 & 620 & 18 & 910 & 0,21 \\
Acier doux & 196000 & 260 & 520 & 28 & 891 & 0,28 \\
\hline
\end{tabular}

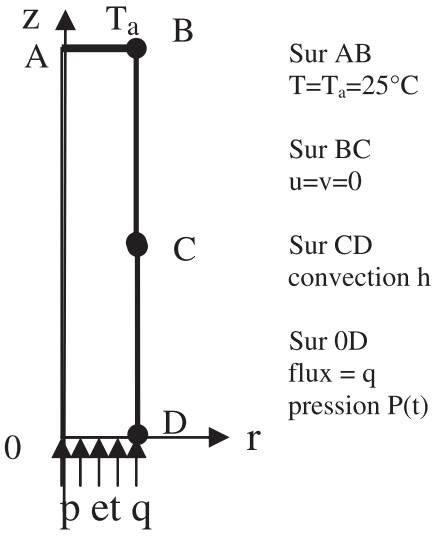

(a)

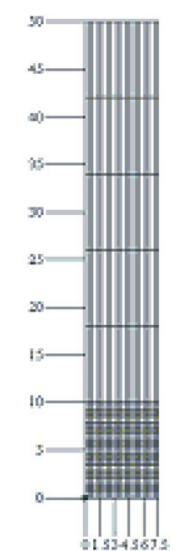

(b)
Fig. 4. a) Conditions aux limites; b) maillage.

en fonction du temps au cours de l'opération de soudage. Les cycles thermiques, relevés en des points situés sur une perpendiculaire à l'interface font apparaître des températures maximales et des vitesses de refroidissement décroissantes au fur et à mesure que l'on s'éloigne de celuici. Cette répartition donne donc lieu à un gradient thermique (Figs. 7 et 8). La propagation de la chaleur dans les pièces est influencée par les propriétés physiques des matériaux, la géométrie, la température initiale des pièces et les conditions d'échange. Le moyen de validation est la mesure de la température en certains points de la zone de soudure. Cette validation est également possible grâce à la comparaison des cycles thermiques expérimentaux avec ceux obtenus par simulation numérique. Il est encore possible de valider par la quantification de certains effets de la température (couleur de la pièce, transformation métallurgique). Pour cette raison nous avons utilisé deux techniques de validation :

i) les isothermes sont caractérisés par une transition de couleur violette $\left(T=450{ }^{\circ} \mathrm{C}\right)$ à la périphérie de l'éprouvette. La chaleur issue de l'interface se propage suivant l'axe de l'éprouvette; le front de la température se propage rapidement puis s'atténue; ensuite il reprend de nouveau avec l'accroissement de la pression de forgeage. La vitesse de propagation de la température dans le modèle numérique est en accord avec celle de l'expérience qui correspond à la troisième séquence du cycle de soudage (phase de forgeage) (Fig. 7) ;

ii) la méthode des coupes macrographiques des soudures visualise la zone affectée thermiquement (Fig. 8).

À l'interface, l'étendue de la zone affectée thermiquement est fortement liée aux paramètres de soudage. 


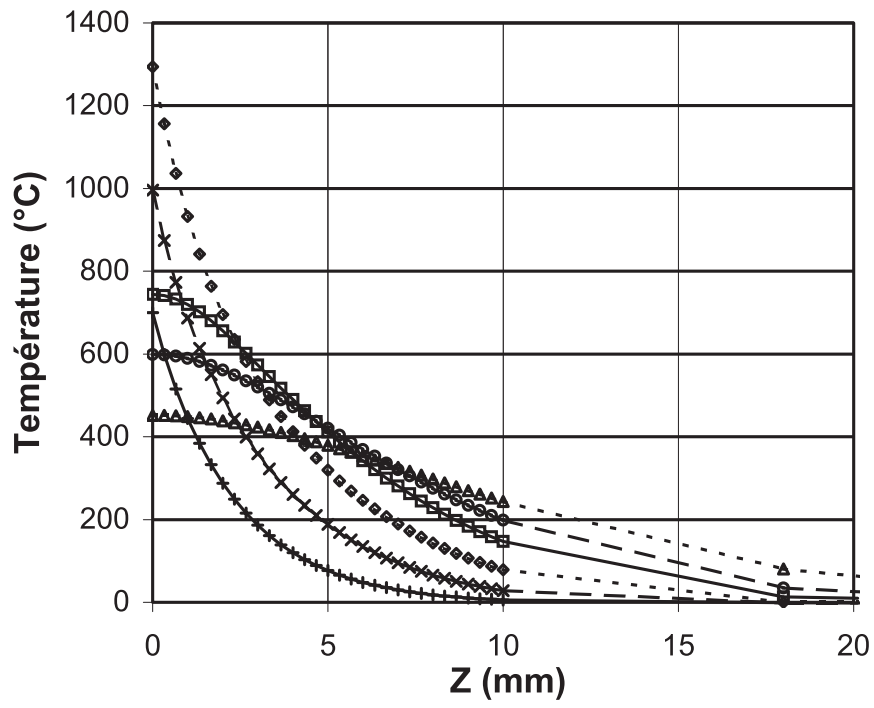

Fig. 5. Distribution de la température à des différents pas de temps suivant l'axe de la pièce.

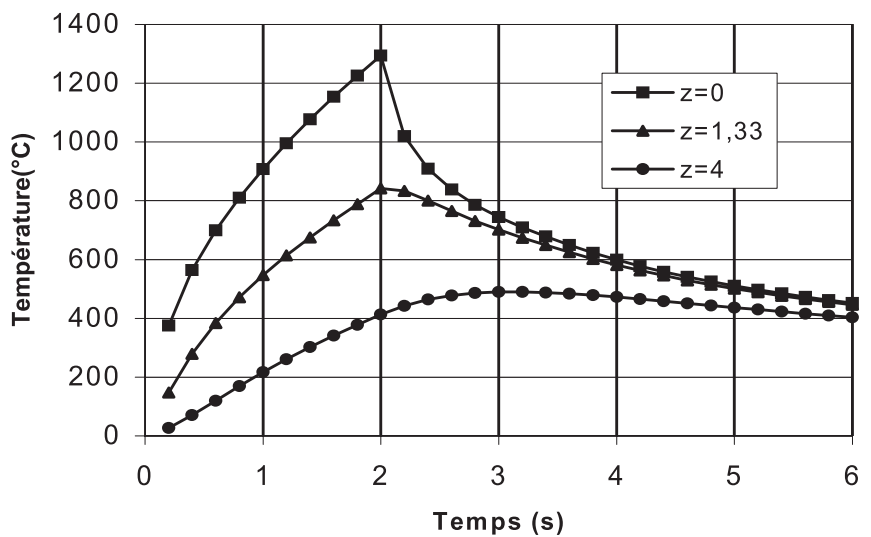

Fig. 6. Cycle thermique de trois positions différentes.

En dépit de leurs simplicités, ces méthodes présentent quelques inconvénients et principalement le manque de précision.

Aux bords de l'interface, le métal subit les effets du cycle thermique de soudage qui provoque des transformations métallurgiques liées à la température maximale atteinte $\theta_{\max }$ et aux conditions de refroidissement du joint soudé. Ces transformations peuvent être synthétisées par la figure 9.

- Au chauffage, le matériau subit des anomalies de dilatation, une plastification et une transformation austénitique (maille $\gamma$ ) jusqu'à $\theta_{\max }$ qui n'atteint pas la température de fusion.

- Au refroidissement, les zones chauffées subissent des transformations qui dépendent essentiellement de la vitesse de refroidissement et du temps de maintien au-dessus de $A_{\mathrm{C} 3}$ (lieu des températures définissant la limite supérieure du domaine d'existence de la ferrite). Le matériau subit alors des contractions et des transformations allotropiques (Martensite ou Martensite + Ferrite ... ). Contrairement au soudage en phase

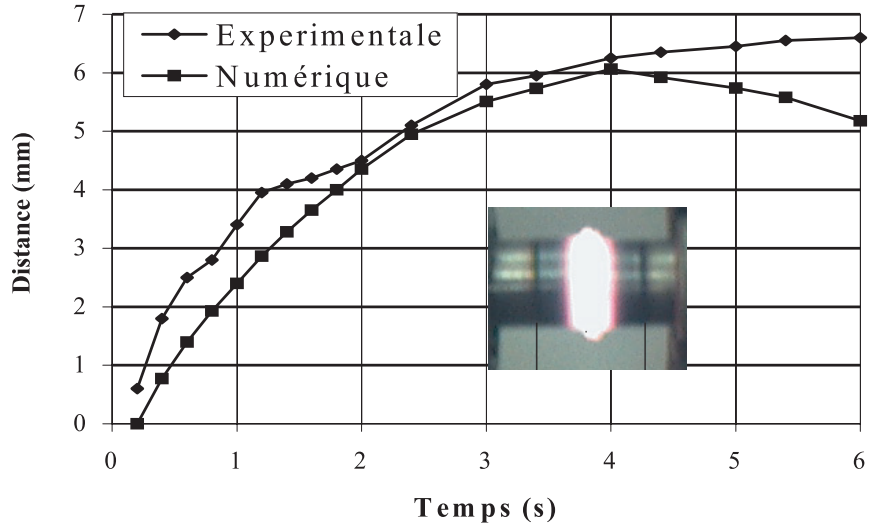

Fig. 7. Zone franchie par $T_{450}{ }^{\circ} \mathrm{C}$ de part et d'autre de l'interface.

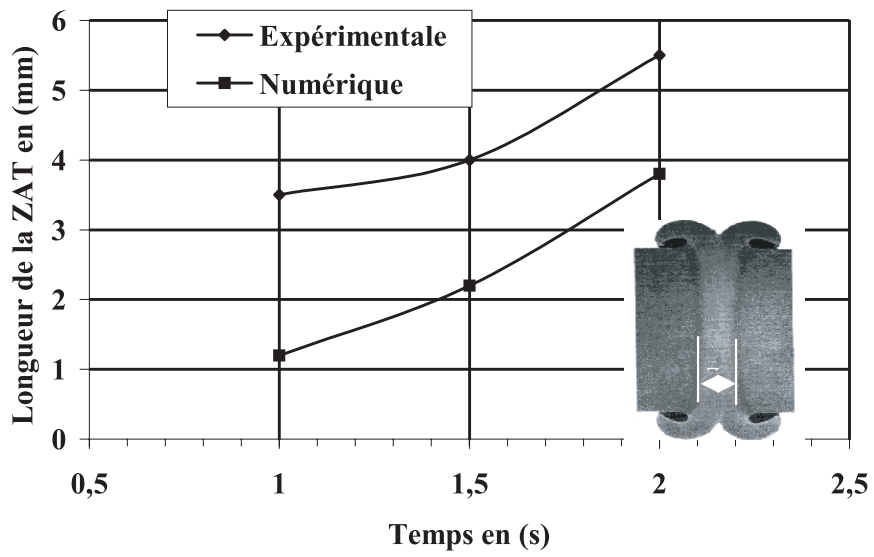

Fig. 8. Évolution de la ZAT en fonction du temps de friction.

liquide, le soudage par friction ne conduit pas à une structure de surchauffe à gros grains qui confère au métal une forte fragilisation après refroidissement. La vitesse de refroidissement influe énormément sur les propriétés mécaniques de l'assemblage. La pression de forgeage va générer dans les grains des contraintes résiduelles au niveau du joint soudé et permettre la rupture par torsion ou par traction plus favorable dans le métal non affecté.

L'expansion du bourrelet en fonction du temps de friction, numérique et expérimentale, est présentée par la figure 10. L'expansion du diamètre est prise après refroidissement pour la courbe expérimentale et instantanément pour la courbe numérique.

\section{Conclusions}

Les phénomènes physiques impliqués dans le soudage par friction sont divers et interdépendants. C'est pourquoi nous nous sommes orientés à étudier quelques aspects phénoménologiques et à résoudre les difficultés que posent l'intégration de chaque mécanisme physique dans une modélisation numérique du procédé. Nous avons aussi procédé à des essais de soudage par friction qui nous ont permis d'étudier l'effet du temps de 




Fig. 9. Cycle thermique et transformations structurales.

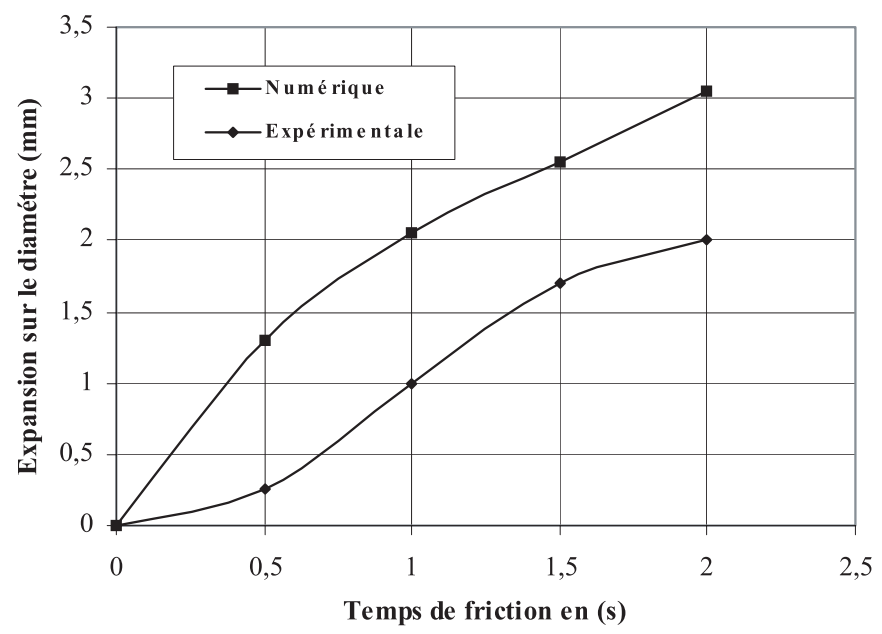

Fig. 10. Expansion du bourrelet en fonction du temps de friction.

friction à la fois sur la propagation de la chaleur et sur la taille de la zone affectée thermiquement. Les résultats expérimentaux obtenus sont en accord avec le modèle numérique développé. Une modélisation de l'aspect énergétique constitue ultérieurement une approche intéressante sur l'optimisation et le contrôle du procédé de soudage par friction.

\section{Annexe A}

\section{Algorithme de plasticité pour le critère de Von Mises et écrouissage isotrope}

- Gradient de déformation et jacobien :

$$
\begin{gathered}
\boldsymbol{F}_{n+1}=\mathbf{1}+\sum_{I=1}^{\text {nnode }} \boldsymbol{u}_{I} \otimes \operatorname{Grad}_{X} N^{I} \\
J_{n+1}=\operatorname{det}\left(\boldsymbol{F}_{n+1}\right), \quad \overline{\boldsymbol{F}}_{n+1}=J_{n+1}^{-1 / 3} \boldsymbol{F}_{n+1}
\end{gathered}
$$

- Prédiction élastique :

$$
\overline{\boldsymbol{b}}^{\mathrm{e}^{T}}=\overline{\boldsymbol{F}}_{n+1} \boldsymbol{C}_{n}^{p^{-1}} \overline{\boldsymbol{F}}_{n+1}^{T}
$$$$
\boldsymbol{s}^{T}=\mu \operatorname{dev}\left(\overline{\boldsymbol{b}}^{\mathrm{e}^{T}}\right), \quad \boldsymbol{n}=\frac{\boldsymbol{s}^{T}}{\left\|\boldsymbol{s}^{T}\right\|}, \quad \bar{\mu}=\frac{\mu}{3} \operatorname{tr}\left(\overline{\boldsymbol{b}}^{\mathrm{e}^{T}}\right)
$$

- Correction plastique :

$$
\sqrt{3 / 2}\left\|s^{T}\right\|-3 \bar{\mu} \Delta p-\sigma_{p}(p, \Theta)=0
$$

- Mise à jour

$$
\begin{gathered}
p_{n+1}=p_{n}+\Delta p \\
\boldsymbol{s}_{n+1}=\boldsymbol{s}^{T}-2 \bar{\mu} \sqrt{3 / 2} \Delta \gamma \boldsymbol{n} \\
\boldsymbol{\tau}_{n+1}=\boldsymbol{s}_{n+1}+J_{n+1} p_{n+1} \mathbf{1}, \quad p_{n+1}=\psi_{\mathrm{vol}}^{\prime}\left(J_{n+1}\right) \\
\overline{\boldsymbol{b}}_{n+1}^{\mathrm{e}}=s_{n+1} / \mu+\frac{1}{3} \operatorname{tr}\left(\overline{\boldsymbol{b}}^{\mathrm{e}^{T}}\right) \mathbf{1} \\
\boldsymbol{C}_{n+1}^{p^{-1}}=\overline{\boldsymbol{F}}_{n+1}^{-1} \overline{\boldsymbol{b}}_{n+1}^{\mathrm{e}} \overline{\boldsymbol{F}}_{n+1}^{-T}
\end{gathered}
$$

- Module tangent

$$
\begin{gathered}
\boldsymbol{c}^{\mathrm{ep}}=\boldsymbol{a}_{\mathrm{vol}}^{T}+\left(1-\delta_{0}\right) \boldsymbol{a}_{\mathrm{dev}}^{T}-\delta_{1} \boldsymbol{n} \otimes \boldsymbol{n}-\delta_{2} \boldsymbol{n} \otimes \operatorname{dev}\left[\boldsymbol{n}^{2}\right] \\
\delta_{0}=\frac{2 \bar{\mu} \sqrt{3 / 2} \Delta \gamma}{\left\|\boldsymbol{s}^{T}\right\|}, \quad \delta_{2}=2\left\|\boldsymbol{s}^{T}\right\|\left(A-\delta_{0}\right) \\
A=\frac{1}{1+\frac{\sigma_{p}^{\prime}}{3 \overline{\bar{\mu}}}} \\
\delta_{1}=2 \bar{\mu}\left(A-\delta_{0}\right)+\frac{4}{3} \sqrt{3 / 2} \Delta \gamma\left\|\boldsymbol{s}^{T}\right\|(1-A)
\end{gathered}
$$

\section{Références}

[1] K. Hollander, Developpements in friction welding, Metals Eng., Quarterly, 1962, p. 1424

[2] C. Laour, Procédé de soudage par friction, Soudage 2000, 14 mars 2001

[3] B. Dufin, The mechanics of friction Welding mild steel, Metal construction, 1976

[4] G. Sayegh, R. Cazes, Possibilités et perspectives du soudage par friction. Application-Évolution de la technologie et perspective, Soudage et techniques connexes, 1981, pp. $152-159$

[5] M. Ayadi, H. Ben Slimane, K. Jendoubi, Influence des paramètres de soudage par friction sur la résistance mécanique des joints soudés, $4^{\text {es }}$ Journées Maghrébines des Sciences des Matériaux, V1, 1994, pp. 53-58

[6] R. Cazes, Procédés de soudage, Principes généraux et critères de choix, Techniques de l'ingénieur B 7, 1981, 745 
[7] L. Primot, Soudage par friction, CETIM Info., 1989, $\mathrm{N}^{\mathrm{o}} 91, \mathrm{~N}^{\mathrm{o}} 105$, pp. $29-45$ et $\mathrm{N}^{\mathrm{o}} 112$ pp. $50-60$

[8] C. Miehe, Entropic thermoelasticity at finite strains. Aspects of the formulation and numerical implementation, Comput. Methods Appl. Mech. Engrg. 120 (1995) 243-269

[9] F. Armero, J.C. Simo, A new unconditionnelly stable fractional step method for nonlinear coupled thermomechanical problems, Internat. J. Numer. Methods Engrg. 35 (1992) 737-766

[10] J.C. Simo, C. Miehe, Associative coupled thermoplasticity at finite strains : Formulation, numerical analysis and implementation, Comput. Methods Appl. Mech. Engrg. 98 (1992) 41-104
[11] T.H. Argyris, L.E. Vaz, J.K. William, Integrated finite element analysis of coupled visoplastic problem, J. Thermal Stresses 4 (1981) 121-153

[12] A. Srikanth, N. Zabaras, A computational model for the finite element analysis of thermoplasticity coupled with damage at finite strains, Internat. J. Numer. Methods Engrg. 45 (1999) 1569-1605

[13] T.J. Oden, Finite Element of Nonlinear continue, McGraw Hill, New York, 1972

[14] H. Granjon, Bases métallurgiques du soudage, I.S. Publication de la soudure autogène, Édition Eyrolles, 1989 